Departamento de Nefrología, Escuela de Medicina. Pontificia Universidad Católica de Chile. Santiago, Chile.

Los autores declaran que no hubo aportes financieros externos en la realización de esta revisión, ni conflictos de intereses. Trabajo no recibió finaciamiento.

Recibido el 19 de octubre de 2017, aceptado el 3 de abril de 2018.

Correspondencia a: Rodrigo Sepúlveda Palamara

Diagonal Paraguay 362 ,

Departamento de Nefrología. $4^{\circ}$ piso.

Teléfono: 56-2-3543229 rrsepulveda@uc.cl

\section{Síndrome hemolítico urémico atípico}

\author{
RODRIGO A. SEPÚLVEDA, RODRIGO TAGLE, AQUILES JARA
}

\section{Atypical hemolytic uremic syndrome}

Atypical hemolytic uremic syndrome (aHUS) is a rare thrombotic microangiopathy, characterized by microangiopathic hemolytic anemia, thrombocytopenia and renal involvement. It causes end stage renal disease requiring dialysis in most affected patients. It mainly affects young adults (contrary to what was thought years ago). When aHUS is primary, the cause is a genetic mutation in the alternative complement pathway. Instead, secondary aHUS is caused by external factors that trigger the disease by themselves or in combination with a genetic vulnerability. The type of mutation determines the severity of the disease, prognosis, response to therapy and renal transplantation. Advances in the understanding of renal diseases associated with complement defects and the development of specific biologic therapies changed the course of this disease. Eculizumab is internationally approved for the treatment of primary aHUS. Its inhibitory action on the complement cascade leads to hematologic remission and restoration of renal function. We present a review of aHUS detailing its etiology, pathogenesis, clinical presentation, diagnosis and treatment.

(Rev Med Chile 2018; 146: 770-779)

Key words: Atypical Hemolytic Uremic Syndrome; Complement System Proteins; Hemolytic-Uremic Syndrome.
$\mathrm{E}$ 1 síndrome hemolítico urémico (SHU) se caracteriza por la tríada de trombocitopenia, anemia hemolítica microangiopática y falla renal aguda. La principal causa es la infección por Escherichia coli productor de toxina Shiga (O157:H7, O111:H8, O103:H2, O104:H4) ${ }^{1}$. La infección por E. coli productora de toxina Shiga ocasiona 90\% de los casos de SHU (SHU-ECTS), siendo una patología principalmente infantil con buen pronóstico ( $>80 \%$ recupera función renal $)^{2}$.

Sin embargo, como existían SHU no producidos por esta infección $(10 \%$ en población infantil), que además se caracterizaban por mal pronóstico, se denominó a estos cuadros SHU atípico $(\mathrm{SHUa})^{3}$.

El SHUa tiene una incidencia anual de 0,5-2 casos por millón de habitantes ${ }^{3}$, siendo la forma más común de SHU en adultos ${ }^{4}$. Sus consecuencias son catastróficas: $5-25 \%$ de mortalidad y $50 \%$ desarrollan enfermedad renal crónica terminal
(ERCT) solo durante la fase aguda, proporción que aumenta en el tiempo ${ }^{5}$.

La patogenia del SHUa corresponde a una microangiopatía trombótica (MAT). MAT es un concepto histopatológico ${ }^{6}$, pero clínicamente se caracteriza por anemia, trombocitopenia y falla orgánica ${ }^{7}$. En el SHU, el compromiso orgánico se limita o predomina en el riñón. El SHUa se caracteriza por una activación anómala del complemento que provoca lesión endotelial, y así, activación de la hemostasia primaria y secundaria.

Como la definición inicial de SHUa fue "SHU no producido por toxina Shiga", se incluyeron causas que no presentaban como evento principal la activación del complemento. Actualmente se considera al SHUa como una patología de la vía alterna del complemento, siendo primario cuando el defecto es genético. Las causas secundarias de SHUa pueden activar el complemento por sí mismas, o actuar como condiciones amplificadoras 
del complemento (CAC) sobre una vulnerabilidad genética (Tabla 1). Es decir, para que se desarrolle una MAT es necesario superar un "umbral" que desencadena activación intravascular de la hemostasia, si esta activación se manifiesta predominantemente con daño renal, será un SHU. Si la causa es exclusivamente mediada por defectos genéticos del complemento el diagnóstico es SHUa primario, si la etiología exclusiva es otra condición (como las CAC), será un SHUa secundario. Sin embargo, este "umbral" de MAT puede lograrse con la suma de defectos genéticos y CAC, que es la forma más frecuente en la práctica clínica (SHUa primario más CAC).

\section{Sistema del complemento}

Conjunto de proteínas plasmáticas sintetizadas en el hígado que participan de la inmunidad innata. Tiene múltiples funciones, como contribuir a la respuesta inflamatoria (anafilotoxinas), opsonización (C3b), eliminación de complejos inmunes y promover la lisis celular mediante la formación del complejo de ataque de membrana
$(\mathrm{CAM})^{8}$. El CAM se produce por 3 vías: clásica, alterna y vía de las lectinas (Figura 1).

Las vías clásica y de las lectinas requieren un estímulo externo para ser activadas. La vía clásica inicia con la unión de $\mathrm{Clq}$ a la porción $\mathrm{Fc}$ de un anticuerpo (IgG o IgM) unido a su respectivo antígeno. Las lectinas son proteínas que se unen a azúcares específicos como residuos de manosa pertenecientes a la superficie de bacterias.

En cambio, la vía alterna tiende a activarse espontáneamente por lo que es necesario un control de esta. Un fallo en sus mecanismos de control producirá SHUa.

El factor C3 del complemento puede sufrir hidrólisis en forma espontánea (C3h) mientras se encuentra en circulación. C3h se asocia al factor $\mathrm{B}$ y factor $\mathrm{D}$, formando el complejo $\mathrm{C} 3 \mathrm{hBb}$. Este último tiene actividad " $\mathrm{C} 3$ convertasa", generando C3a y C3b. El primero actúa como anafilotoxina, el segundo se adhiere a membranas celulares (endotelio, bacterias, etc.). C3b también se asocia a los factores $\mathrm{B}$ y $\mathrm{D}$, conformándose el complejo $\mathrm{C} 3 \mathrm{bBb}$ en las membranas celulares. Este último complejo posee actividad C3 convertasa y amplifica la reacción. Cuando C3b se une al complejo

Tabla 1. Causas de Síndrome Hemolítico Urémico atípico

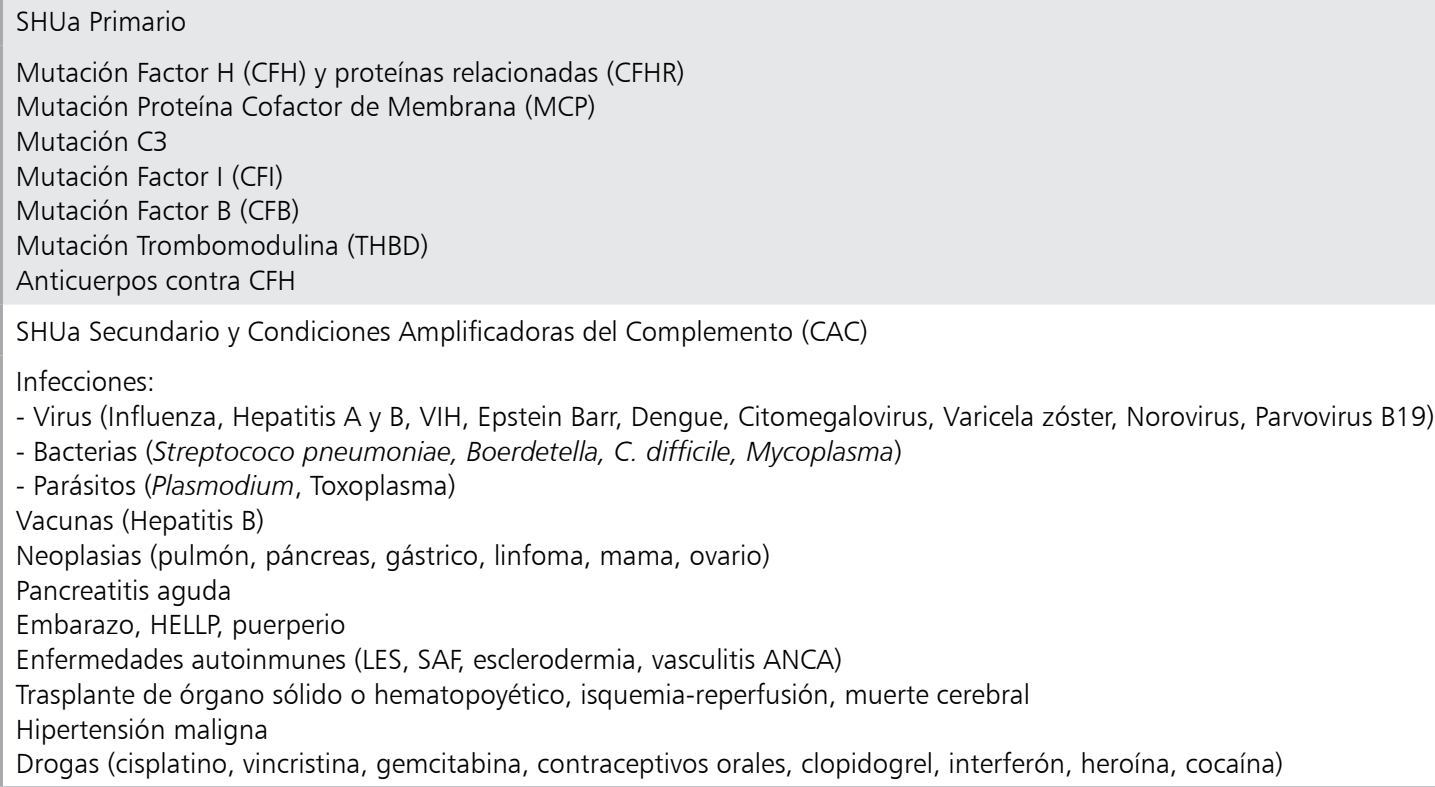

ANCA: anticuerpos anticitoplasma de neutrófilos; HELLP: hemólisis, elevación enzimas hepáticas, bajas plaquetas; LES: lupus eritematoso sistémico; SAF: síndrome antifosfolípidos; VIH: virus de la inmunodeficiencia humana. 


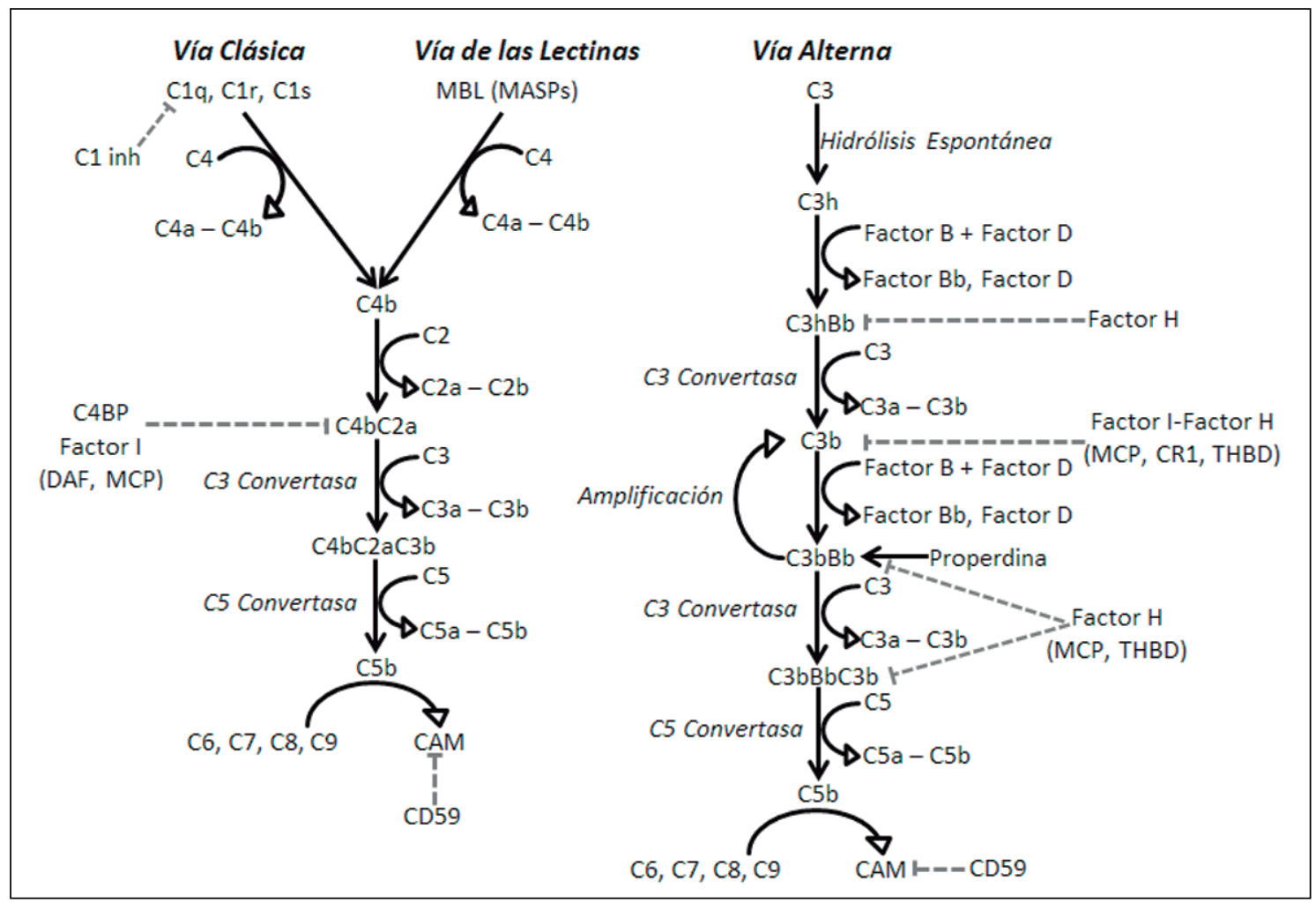

Figura 1. Sistema del complemento. $C 1$ inh: inhibidor de C1; C4BP: proteína de unión a C4; CR1: receptor del complemento tipo1; CAM: complejo de ataque de membrana; DAF: factor acelerador del decaimiento; MASP: proteína esterasa asociada a MBL; MCP: proteína cofactor de membrana; MBL: lectina de unión a manosa; THBD: trombomodulina.

$\mathrm{C} 3 \mathrm{bBb}$ forma $\mathrm{C} 3 \mathrm{bBbC} 3 \mathrm{~b}$ que tiene actividad " $\mathrm{C} 5$ convertasa". Finalmente, será este último el que genere C5a (anafilotoxina) y C5b, el cual producirá la formación del CAM con lisis celular.

Para prevenir el daño celular mediado por el complemento existen inhibidores plasmáticos: $\mathrm{CFH}, \mathrm{CFI}, \mathrm{C} 4 \mathrm{BP}$ y el inhibidor de C1. Además, existen inhibidores en las membranas celulares: factor acelerador del decaimiento (DAF), receptor de C1 (CR1), CD59, MCP y THBD. Tanto el CFH, como MCP, THBD, C4BP, DAF y C1 inhibidor desestructuran los complejos que se forman durante la cascada del complemento (aceleración del decaimiento). El CFI escinde C3b y C4b en presencia de MCP y CFH. El CFH inactiva el complejo $\mathrm{C} 3 \mathrm{bBb}$ (C3 convertasa) y C3bBbC3b (C5 convertasa) uniéndose a sustancias polianiónicas de la membrana plasmática o matriz extracelular. Los inhibidores de membranas actúan como co- factores para que los reguladores plasmáticos se unan a sus respectivos complejos (adheridos a la membrana). La presencia de cofactores (inhibidores de membrana) es fundamental; así CFH solo se une a los complejos adheridos a membranas celulares propias y no a las de células externas, permitiendo que actúe el complemento ${ }^{8}$.

La properdina es una proteína producida por neutrófilos, su función es estabilizar el complejo $\mathrm{C} 3 \mathrm{bBb}$ (intrínsecamente inestable) e inhibir al $\mathrm{CFH}$ para favorecer la vía alterna del complemento ${ }^{8}$.

Por lo tanto, son múltiples las alteraciones de la vía alterna que pueden provocar SHUa primario; actualmente se conocen modificaciones en $\mathrm{CFH}$, CFHR (proteínas relacionadas al CFH), CFI, MCP, THBD y mutaciones activantes de C3 y CFB.

El glomérulo carece de matriz extracelular (rico en sustancias polianiónicas) y de agentes 
protectores de membrana (DAF, CD59 y THBD), motivo por el cual es más vulnerable a la activación del complemento ${ }^{9}$.

\section{Cuadro clínico}

El SHUa se caracteriza por una tríada: anemia hemolítica microangiopática no inmunológica, trombocitopenia y falla renal aguda. Diferentes patologías tienen presentación similar: SHU-ECTS, púrpura trombocitopénico trombótico (PTT) y otras MAT no mediadas por activación primaria del complemento.

El cuadro clínico es brusco, pero en $20 \%$ se manifiesta insidiosamente: anemia subclínica, trombocitopenia fluctuante y función renal preservada. Presentaciones atípicas se caracterizan por ausencia de trombocitopenia (15\%), ausencia de anemia $(6 \%)$, preservación de la función renal (17\%), síndrome nefrótico o hipertensión arterial maligna. La trombocitopenia raramente es grave y el riesgo de sangrado es mínimo ${ }^{4,10-12}$.

El grupo etario afectado por SHUa es controversial; se describía que predominaba en menores de 18 años, aunque $12-50 \%$ de los casos ocurre en mayores de 25 años (incluso hasta 83 años) ${ }^{11}$. Otras cohortes lo clasifican como una enfermedad propia de adultos (58\% de los casos) ${ }^{13}$. La relación mujer:hombre es 1:1 en población infantil, pero en adultos predomina en mujeres. El 70\% de los infantes presentan su primer episodio antes de los 2 años. Un SHU en lactantes menores de 12 meses es sugerente de $\mathrm{SHUa}^{5}$.

En SHUa primario se documenta un evento gatillante en $39-70 \%$, siendo lo más frecuente infecciones gastrointestinales, respiratorias y embarazo/puerperio ${ }^{10}$. Una MAT o un síndrome HELLP durante el embarazo y puerperio, frecuentemente tienen de base un SHUa ${ }^{4,7}$.

La afección extrarrenal ocurre en 20\% de los casos y los órganos más comprometidos son: corazón, intestino, páncreas, pulmones y encéfalo ${ }^{10}$. Hasta $30 \%$ puede presentarse con diarrea, por lo que no es útil para diferenciar con SHU-ECTS ${ }^{3}$. Los niveles de C3 son bajos solo en 36\% de los casos $^{10}$.

Luego de un primer cuadro, el riesgo de recaída es $30 \%$ en adultos, incluyendo los que no tienen mutación demostrable. De los pacientes que recurren, $90 \%$ lo hace el primer año y posterior a este período el riesgo baja a $20-25 \%{ }^{12}$.

El pronóstico es malo, 59\% de los niños y $81 \%$ de los adultos requieren diálisis al momento de presentación y la mortalidad, en el período agudo, se ha descrito en $0,8 \%$ para adultos y $6,7 \%$ en infantes. Al cabo de 3 a 5 años, 50\% de los infantes y $70 \%$ de los adultos estarán muertos o dependientes de diálisis ${ }^{10,14}$.

\section{Genética en SHUa}

En $60-70 \%$ de los SHUa primarios es detectable una alteración genética, en el resto solo es presunta ${ }^{10}$. Los casos esporádicos tienen mejor pronóstico que los familiares. La penetrancia general es $50 \%{ }^{5}$. En pacientes menores de 1 año predominan mutaciones $\mathrm{CFH}, \mathrm{CFI}$, THBD y C3; luego del año se agrega la mutación MCP. Entre los 7 y 11 años son característicos los anticuerpos anti-CFH ${ }^{4}$. En la Tabla 2 se mencionan las principales características de cada mutación.

\section{Enfrentamiento diagnóstico del SHUa}

El SHU se sospecha al objetivar la tríada de trombocitopenia, anemia hemolítica microangiopática y falla renal aguda.

En pacientes con MAT habrá trombocitopenia (plaquetas $<150.000 / \mu \mathrm{L}$ o descenso $>25 \%$ respecto al basal) y hemólisis microangiopática (hemoglobina $<10 \mathrm{~g} / \mathrm{dL}$, Coombs negativo, $\mathrm{LDH}$ elevada, haptoglobina baja, reticulocitosis, hemoglobinuria o presencia de esquistocitos). Una proporción de esquistocitos $>1 \%$ es diagnóstico de MAT en ausencia de otra causa ${ }^{15}$.

El diagnóstico de SHUa primario en contexto de MAT es de exclusión. Será fundamental buscar CAC (Tabla 1) y descartar otras etiologías de MAT que se manifiestan como SHU pero no son mediadas por alteraciones del complemento: drogas, neoplasias, infecciones, PTT, SHU-ECTS y déficit de cobalamina $\mathrm{C}$ (Tabla 3 ). El embarazo se asocia con PTT y el puerperio a SHUa.

Los principales diagnósticos diferenciales son PTT y SHU-ECTS. El primero se corrobora con medición de actividad de metaloproteinasas $<5-10 \%$, y el segundo con cultivos en deposiciones o búsqueda de toxina Shiga. Hasta 30\% de los casos de SHU-ECTS puede tener estudio 
Tabla 2. Mutaciones SHUa primario

\begin{tabular}{|c|c|c|c|c|c|c|c|c|}
\hline Mutación & CFH & CFHR & MCP & CFI & C3 & THBD & Ac anti-CFH & $\underset{\text { Sin }}{\text { mutación }}$ \\
\hline Frecuencia & $20-30 \%$ & $3-10 \%$ & $8-10 \%$ & $6 \%$ & $6 \%$ & $<5 \%$ & $10 \%$ & $30 \%$ \\
\hline $\begin{array}{l}\text { Respuesta a } \\
\text { plasmaterapia }\end{array}$ & $63 \%$ & & $0 \%$ & $25 \%$ & $57 \%$ & $88 \%$ & $75 \%$ & $50 \%$ \\
\hline $\begin{array}{l}\text { Diálisis/muerte } \\
\text { a } 5 \text { años }\end{array}$ & $77 \%$ & & $35 \%$ & $65 \%$ & $65 \%$ & $54 \%$ & $60 \%$ & \\
\hline $\begin{array}{l}\text { Recurrencia Tx } \\
\text { Renal }\end{array}$ & $70-85 \%$ & & $10-20 \%$ & $50 \%$ & $80 \%$ & Alta & $\begin{array}{l}\text { Según títulos } \\
\text { anticuerpos }\end{array}$ & $20 \%$ \\
\hline Otros & $\begin{array}{c}\text { Mal pronóstico } \\
\text { Alta incidencia } \\
\text { de enfermedades } \\
\text { cardiovasculares }\end{array}$ & $\begin{array}{l}\text { Similar a } \\
\text { mutación } \\
\text { CFH }\end{array}$ & $\begin{array}{l}\text { Recurrencias } \\
\text { frecuentes, } \\
\text { pero buen } \\
\text { pronóstico }\end{array}$ & & $\begin{array}{c}\text { Mal } \\
\text { pronóstico }\end{array}$ & & $\begin{array}{c}\text { Deleción } \\
\text { homocigota } \\
\text { CFHR1,3 }\end{array}$ & $\begin{array}{c}\text { Mejor } \\
\text { pronóstico }\end{array}$ \\
\hline
\end{tabular}

Ac: anticuerpos, CFH: factor $\mathrm{H}$ del complemento, CFHR: proteínas relacionados al CFH, CFI: factor I del complemento, THBD: trombomodulina, Tx: trasplante.

\section{Tabla 3. Estudios etiológicos recomendados}

\begin{tabular}{|c|c|}
\hline $\begin{array}{l}\text { Infección por E. coli } \\
\text { productora de toxina }\end{array}$ & $\begin{array}{l}\text { - Muestra fecal obtenida desde diarrea o hisopado rectal para cultivos (incluyendo medio } \\
\text { MacConkey para E. coli } 0157: \mathrm{H7} \text { ) }\end{array}$ \\
\hline Shiga & $\begin{array}{l}\text { - PCR para gen de verotoxina y/o enteropatógenos específicos } \\
\text { - ELISA para verotoxina }\end{array}$ \\
\hline $\begin{array}{l}\text { Infección por } \\
\text { neumococo productor de } \\
\text { neuraminidasa }\end{array}$ & $\begin{array}{l}\text { - Cultivos bacterianos } \\
\text { - Antígeno urinario de S. pneumoniae } \\
\text { - Coombs directo falso (+) } \\
\text { - Radiografía de tórax (característicamente presenta derrame pleural) }\end{array}$ \\
\hline $\begin{array}{l}\text { Alteraciones en } \\
\text { la regulación del } \\
\text { complemento }\end{array}$ & $\begin{array}{l}\text { - Niveles de C3, C4, CFH, CFI, CFB, CH50, AH50 (medir previo a plasmaterapia) } \\
\text { - Mutaciones en CFH, CHFR, CFI, CFB, MCP, THBD } \\
\text { - Autoanticuerpos anti-CFH (medir previo a plasmaterapia) } \\
\text { - Expresión leucocitaria de MCP }\end{array}$ \\
\hline $\begin{array}{l}\text { Metabolismo de } \\
\text { cobalamina }\end{array}$ & $\begin{array}{l}\text { - Cromatografía de aminoácidos en plasma (hiperhomocisteinemia, hipometioninemia) } \\
\text { - Cromatografía de ácidos orgánicos en orina (aciduria metilmalónica) } \\
\text { - Mutación del gen MMACHC }\end{array}$ \\
\hline Infecciones virales & $\begin{array}{l}\text { - PCR y/o ELISA para VIH } \\
\text { - PCR y/o IFI para virus Influenza }\end{array}$ \\
\hline Embarazo, HELLP & $\begin{array}{l}\text { - Test de embarazo, } \beta \text {-HCG } \\
\text { - Enzimas hepáticas, uricemia }\end{array}$ \\
\hline $\begin{array}{l}\text { Enfermedades } \\
\text { autoinmunes }\end{array}$ & $\begin{array}{l}\text { - Anticuerpos antinucleares } \\
\text { - Estudio de SAF (anticuerpos anti-cardiolipinas, anti- } \beta \text {-2-glicoproteína-1, anticoagulante } \\
\text { lúpico) }\end{array}$ \\
\hline
\end{tabular}

ELISA: enzyme linked immunosorbent assay; HELLP: hemólisis, elevación enzimas hepáticas, plaquetas bajas; IFI: inmunofluorescencia indirecta; MMACHC: aciduria metilmalónica y homocistinuria tipo C; SAF: síndrome antifosfolípidos; SHUa: síndrome hemolítico urémico atípico; VIH: virus de la inmunodeficiencia humana. 
etiológico negativo y muchas veces el diagnóstico dependerá de la presunción clínica ${ }^{11}$. La presencia de creatinina $<2,27 \mathrm{mg} / \mathrm{dL}$ más recuento plaquetario $<30.000 / \mu \mathrm{L}$, es altamente predictivo de $\mathrm{PTT}^{16}$. Con el diagnóstico de MAT se recomienda iniciar plasmaféresis, ya que una etiología posible es el PTT, cuyo mal pronóstico cambia drásticamente con plasmaterapia. Una vez descartando PTT y SHU-ECTS, se puede asumir SHUa y manejarlo como tal. Previo a la plasmaterapia, es necesario obtener una muestra para evaluación de
C3, C4, CFH, CFI, CFB, CH50, AH50, anticuerpos anti-CFH y actividad de metaloproteinasa. Estos exámenes serán fundamentales en el diagnóstico diferencial y etiológico del SHUa (Figura 2).

Se recomienda realizar el estudio genético en todos los pacientes con SHUa presunto o en aquellos donde exista una CAC agregada ${ }^{11}$. Permite confirmar una enfermedad dependiente del complemento, establecer pronóstico, tratamiento, riesgo de recurrencias, progresión a ERCT, consejo genético familiar y ayuda en la decisión de tras-

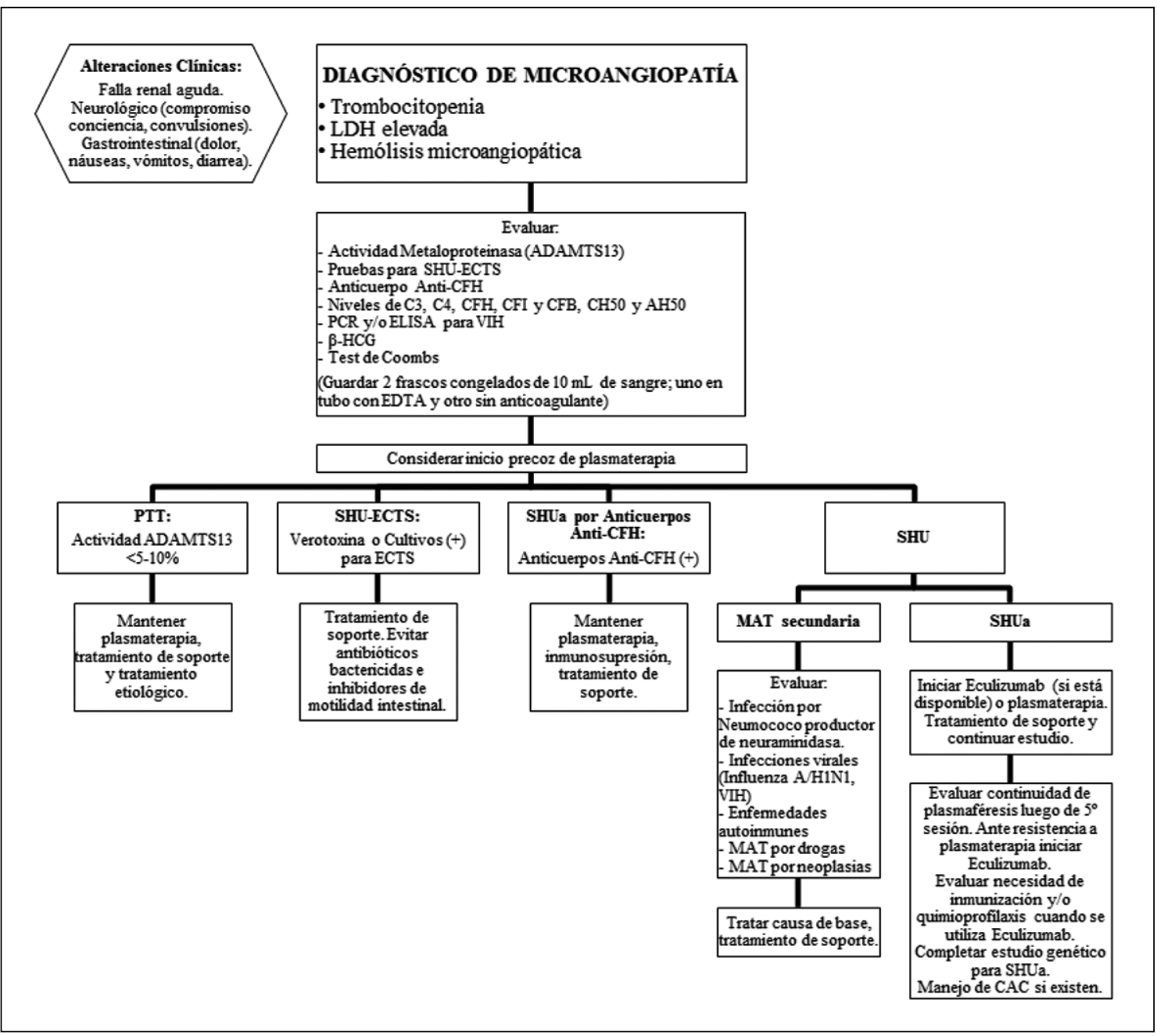

Figura 2. Enfrentamiento propuesto para diagnóstico y manejo de SHU. ADAMTS13: A disintegrin-like and metalloprotease with thrombospondin type 1 motif no. 13; ANA: anticuerpos antinucleares; $\beta$-HCG: gonadotrofina coriónica humana $\beta$; CAC: condiciones amplificadoras del complemento; EDTA: ácido etilendiaminotetraacético; LDH: lactato deshidrogenasa; MAT: microangiopatía trombótica; PTT: púrpura trombocitopénico trombótico; SAF: síndrome antifosfolípidos; SHU: síndrome hemolítico urémico; SHUa: SHU atípico; SHU-ECTS: SHU producido por E. coli productor de toxina Shiga; VIH: virus de la inmunodeficiencia humana. 
plante renal. No es necesario en MAT secundario a drogas, cáncer, infecciones por S. pneumoniae, virus Influenza, VIH, déficit de C-cobalamina, PTT, ni SHU-ECTS. El estudio genético no es una prioridad, es posible diferirlo y prescindir de sus resultados para el manejo inicial.

La biopsia renal corrobora una MAT y aporta datos de pronóstico, sin embargo, no es necesaria para el diagnóstico y se debe considerar que estos pacientes tienen mayor riesgo de sangrado que otras nefropatías.

\section{Tratamiento}

Una vez establecido el diagnóstico de SHUa, además de entregar todas las medidas de soporte incluyendo diálisis si se requiere; es necesario tratar la alteración en la vía alterna. En casos de MAT secundaria, el retiro del agente causal permite la regresión del cuadro.

\section{Tratamiento de soporte}

Los pacientes con MAT y SHUa deben ser tratados en centros especializados. Tratar la falla renal aguda y sus complicaciones.

La transfusión de plaquetas está contraindicada en SHUa porque acentúa fenómenos trombóticos, excepto que el paciente sangre activamente o cuando se realice un procedimiento quirúrgico $y$ el recuento plaquetario sea $<30.000 / \mu \mathrm{L}$.

Proteger una extremidad superior de punciones y evitar el uso de accesos venosos centrales subclavios, ante la alta probabilidad de necesitar diálisis en forma indefinida.

\section{Plasmaterapia}

Hasta el año 2010, consistía en el tratamiento de primera línea para SHUa. La utilidad de la plasmaféresis nunca ha sido estudiada directamente, pero se estima que reduce la mortalidad ${ }^{1}$.

Las terapias de plasma son útiles cuando el defecto se debe a un factor circulante, pero no habrá respuesta si la alteración se encuentra en la membrana plasmática. Pacientes con mutaciones activantes pueden empeorar por la adición de sustratos, sin embargo, cuando se agrega aféresis, estos son extraídos 9 . Cincuenta por ciento de los pacientes con SHUa primario responden a plasmaterapia, pero sólo $20 \%$ de los asociados al embarazo/puerperio ${ }^{7}$. No está claro si la plasmaféresis es más efectiva que la infusión de plasma aislada, pero podría tener beneficios en mutaciones activantes, presencia de autoanticuerpos y sobrecarga de volumen. También se ha visto mayor efectividad en mutación del $\mathrm{CFH}^{4,9}$.

Para SHUa se recomienda plasmaféresis diarias (al menos 5 días) con intercambio de 1-2 volemias en adultos y $100 \mathrm{~mL} / \mathrm{kg}$ en infantes. La reposición se debe realizar con plasma fresco congelado (PFC), ya que es terapéutico en PTT y $\mathrm{SHUa}^{17}$. Cuando no existe disponibilidad de plasmaféresis, se sugiere PFC 10-20 mL/kg.

La respuesta se evalúa a la quinta sesión, específicamente estabilización o mejoría en recuentos hematológicos y grado de daño orgánico. Resistencia a plasmaféresis se define como la imposibilidad de mejorar los recuentos plaquetarios, o en reducir niveles de $\mathrm{LDH}$, o disminuir la creatinina sérica al menos en 25\%; todo esto luego de 5 o más sesiones de plasmaféresis diarias ${ }^{17}$. Cuando existe respuesta (mejoría en los recuentos plaquetarios y ausencia de progresión en el daño orgánico) se debe mantener plasmaféresis diarias hasta la normalización de recuentos plaquetarios y LDH. Luego se recomienda continuar plasmaterapia disminuyendo la frecuencia de estas hasta un esquema de tratamiento mensual, pudiendo mantenerse por varios años. Si la plasmaféresis no entrega beneficios debe ser descontinuada por sus complicaciones.

\section{Eculizumab}

Anticuerpo monoclonal humanizado recombinante contra la fracción C5 del complemento, al unirse evita su clivaje. Combina en su estructura IgG2/IgG4 para evitar la unión a receptores Fc y complemento. Su vida media es 11-12 días y el paso transplacentario es mínimo ${ }^{12}$. Niveles de eculizumab $>100 \mu \mathrm{g} / \mathrm{mL}$ logran un bloqueo completo del complemento a la primera hora de administración ${ }^{18} \mathrm{y}$ a partir de esto se protocolizaron las dosis recomendadas actualmente (Tabla 4). La monitorización se puede realizar con evaluaciones predosis de niveles plasmáticos del fármaco y/o CH50 (buscando un valor $<10 \%$ del esperado ${ }^{19}$ ).

En el año 2011 se aprobó eculizumab como tratamiento de SHUa por la FDA (Food and Drug Administration de los Estados Unidos de Norteamérica) y EMA (European Medicines Agency). Produce respuesta favorable en $85 \%$ de los pacientes resistentes a plasmaféresis o dependientes 
Tabla 4. Dosis eculizumab

\begin{tabular}{|cll|}
\hline Peso del paciente & Dosis inducción & Dosis mantención \\
\hline $40 \mathrm{Kg}$ & $900 \mathrm{mg}$ semanales $\times 4$ dosis & $1.200 \mathrm{mg}$ en la semana 5, luego 1.200 mg cada 2 semanas \\
\hline $30-40 \mathrm{Kg}$ & $600 \mathrm{mg}$ semanales $\times 2$ dosis & $900 \mathrm{mg}$ en la semana 3, luego $900 \mathrm{mg}$ cada 2 semanas \\
\hline $20-30 \mathrm{Kg}$ & $600 \mathrm{mg}$ semanales $\times 2$ dosis & $600 \mathrm{mg}$ en la semana 3, luego $600 \mathrm{mg}$ cada 2 semanas \\
\hline $10-20 \mathrm{Kg}$ & $600 \mathrm{mg}$ semanales $\times 1$ dosis & $300 \mathrm{mg}$ en la semana 2, luego 300 mg cada 2 semanas \\
\hline $5-10 \mathrm{Kg}$ & $300 \mathrm{mg}$ semanales $\times 1$ dosis & $300 \mathrm{mg}$ en la semana 2, luego 300 mg cada 2 semanas \\
\hline
\end{tabular}

de esta, además, es útil en SHUa primario con o sin mutación demostrada ${ }^{18}$.

Se recomienda eculizumab como tratamiento de primera línea en SHUa. Comenzar dentro de 24-48 $\mathrm{h}$ posteriores al ingreso del paciente, si no, iniciar plasmaterapia (idealmente plasmaféresis) y cambiar prontamente a eculizumab.

El riesgo de infección por Neisseria meningitidis en tratamiento con eculizumab es 4 casos por 1.000 pacientes/año ${ }^{12}$. Se recomienda inmunización con vacuna cuadrivalente (ACWY) conjugada y vacuna contra serotipo B. Los títulos protectores se logran a las 2 semanas después de la primera dosis con vacuna cuadrivalente y 1 mes con vacuna serotipo B. Debe administrarse quimioprofilaxis cuando no se realiza vacunación o durante períodos de ventana (amoxicilina o ciprofloxacino por $1 \mathrm{mes})^{20}$. Luego administrar un booster de vacuna cuadrivalente (ACWY) cada 2-5 años o según títulos de anticuerpos (no definido para vacuna contra serotipo B ${ }^{20,21}$. Suspender quimioprofilaxis 60 días después de finalizado tratamiento con eculizumab ${ }^{11}$. Se recomienda monitorización anual de títulos de anticuerpos contra meningococo. También se recomienda vacunar contra Streptococcus pneumoniae y Haemophilus influenzae tipo B; y educar a pacientes sexualmente activos sobre el riesgo de infecciones gonocócicas diseminadas y tratarlas si están presentes ${ }^{12}$.

Las infecciones son condiciones amplificadoras del complemento y gatillantes de SHUa, por lo que se deben prevenir y mantener eculizumab durante estas; incluso es planteable el aumento de dosis y monitorización con niveles plasmáticos o $\mathrm{CH} 50$.

Los ensayos clínicos ${ }^{18,22}$ demuestran que eculizumab permite obtener remisión hematológica en $90 \%$ de los pacientes, produciéndose mejoría del recuento plaquetario durante los primeros días de tratamiento y el máximo aumento de hemoglobina cercano a la $26^{\circ}$ semana. La remisión hematológica se producirá independiente del momento de inicio del fármaco. Sin embargo, la mejoría renal depende de la precocidad del tratamiento, siendo nula cuando se instaura en fase crónica-estable (daño renal ya está establecido) y significativa cuando se inicia en fase aguda-activa (daño renal reversible). La mejoría de la función renal en fase aguda puede ser completa si la terapia se inicia en las primeras horas ${ }^{23}$. La mantención del tratamiento inhibe la vía alterna, evitando la alteración hematológica y nuevo daño renal, pero no podrá reparar el daño renal crónico establecido.

Una vez lograda la remisión, no se recomienda prolongar el intervalo entre dosis, ya que genera períodos sin bloqueo del complemento.

La duración del tratamiento es controversial, al menos, el tratamiento debiera mantenerse durante el período de mayor riesgo, el cual se produce dentro del primer año de un primer episodio. Se recomienda tratamiento indefinido en mutaciones de mal pronóstico (CFH, CFHR, C3 y CFB), trasplante renal, recaídas frecuentes y cuando hubo riesgo vital por $\mathrm{SHUa}^{12,17,24}$.

\section{Trasplante renal}

En adultos, luego de 5 años de trasplante, $42 \%$ requerirá nuevamente diálisis y $7 \%$ estará muerto. La recurrencia de SHUa ocurre en $60-70 \%$ de los pacientes ${ }^{14}$. El $43 \%$ de las recurrencias ocurren en el primer mes postrasplante y $70 \%$ durante el primer año, con un tiempo promedio de pérdida del injerto de 6 meses $^{25}$. El riesgo de pérdida del injerto con una primera recurrencia es $80-90 \%{ }^{4}$.

El trasplante renal proporciona un medio 
idóneo para la reactivación de SHUa. La muerte encefálica aumenta C5a y la isquemia degrada el glicocálix endotelial impidiendo la unión protectora del $\mathrm{CFH}^{26}$.

Los anticalcineurínicos producen MAT postrasplante. Fármacos inhibidores mTOR también se han asociado a esta complicación y con recurrencia de $\mathrm{SHUa}^{27}$. Se recomienda no usar inhibidores mTOR. Betalacept podría ser una alternativa de inmunosupresión sin riesgos de MAT. El uso de anticalcineurínicos no está contraindicado, pero se debe evitar sobreexposición. Estos fármacos no aumentan directamente el riesgo de recurrencia de SHUa, ya que producen microangiopatía por toxicidad endotelial ${ }^{7}$.

Si se considera el trasplante renal con donante vivo, será fundamental el estudio genético del donante ${ }^{11}$. Evitar el trasplante renal sin previo estudio genético del receptor, ya que el pronóstico varía ampliamente según el tipo de mutación (Tabla 2). No se recomienda el trasplante con criterios de donante expandido, isquemia prolongada, donante fallecido por paro cardiaco, crossmatches positivos o con anticuerpos donante-específicos ${ }^{21}$.

La administración de eculizumab ${ }^{28}, \mathrm{PFC}^{29} \mathrm{o}$ plasmaféresis ${ }^{30}$ durante y posterior al trasplante disminuyen recurrencia de SHUa. La duración del tratamiento preventivo con eculizumab o plasmaterapia no se ha establecido, pero probablemente deba ser permanente ${ }^{21}$.

\section{Tratamiento SHUa por anticuerpos anti-CFH}

En el período agudo requiere tratamiento con plasmaféresis e inmunosupresión con corticoides más ciclofosfamida o rituximab ${ }^{11}$. Títulos de anticuerpos $\leq 1.000 \mathrm{AU} / \mathrm{mL}$ se asocian a remisión hematológica y resultados favorables. El tratamiento de mantención continuará con corticoides asociados a micofenolato o azatioprina. El objetivo es mantener títulos $\leq 2.000 \mathrm{AU} / \mathrm{mL}$ ya que sobre este valor aumenta significativamente la probabilidad de recurrencia ${ }^{31}$.

\section{Trasplante hígado-riñón}

El hígado sintetiza los factores del complemento y proteínas reguladoras circulantes, por lo tanto, produce curación del SHUa. Sin embargo, la isquemia y reperfusión inherentes al trasplante activan el complemento. En centros con experiencia, la mortalidad por el procedimiento quirúrgico es $15 \%{ }^{10}$. La decisión de un trasplante combinado debe ser tomada caso a caso y luego de una valoración riesgo-beneficio.

\section{Referencias}

1. Noris M, Remuzzi G. Atypical Hemolytic-Uremic Syndrome. N Engl J Med 2009; 361 (17): 1676-87.

2. Noris M, Mescia F, Remuzzi G. STEC-HUS, atypical HUS and TTP are all diseases of complement activation. Nat Rev Nephrol 2012; 8 (11): 622-33.

3. Noris M, Remuzzi G. Glomerular Diseases Dependent on Complement Activation, Including Atypical Hemolytic Uremic Syndrome, Membranoproliferative Glomerulonephritis, and C3 Glomerulopathy: Core Curriculum 2015. Am J Kidney Dis 2015; 66 (2): 359-75.

4. Loirat C, Frémeaux-Bacchi V. Atypical hemolytic uremic syndrome. Orphanet J Rare Dis 2011; 6 (60): 1-30.

5. Noris M, Caprioli J, Bresin E, Mossali C, Pianetti G, Gamba S, et al. Relative Role of Genetic Complement Abnormalities in Sporadic and Familial aHUS and Their Impact on Clinical Phenotype. Clin J Am Soc Nephrol 2010; 5 (10): 1844-59.

6. George JN, Nester CM. Syndromes of thrombotic microangiopathy. N Engl J Med 2014; 371 (7): 654-66.

7. Riedl M, Fakhouri F, Le Quintrec M, Noone DG, Jungraithmayr TC, Fremeaux-Bacchi V, et al. Spectrum of complement-mediated thrombotic microangiopathies: pathogenetic insights identifying novel treatment approaches. Semin Thromb Hemost 2014; 40 (4): 44464.

8. Ferreira VP, Pangburn MK, Cortés C. Complement control protein factor $\mathrm{H}$ : the good, the bad, and the inadequate. Mol Immunol 2010; 47 (13): 2187-97.

9. De Vriese AS, Sethi S, Van Praet J, Nath KA, Fervenza FC. Kidney Disease Caused by Dysregulation of the Complement Alternative Pathway: An Etiologic Approach. J Am Soc Nephrol 2015; 26 (12): 2917-29.

10. Nester CM, Barbour T, de Cordoba SR, Dragon-Durey MA, Fremeaux-Bacchi V, Goodship TH, et al. Atypical aHUS: State of the art. Mol Immunol 2015; 67 (1): 3142.

11. Loirat C, Fakhouri F, Ariceta G, Besbas N, Bitzan M, Bjerre A, et al. An international consensus approach to the management of atypical hemolytic uremic syndrome in children. Pediatr Nephrol 2016; 31 (1): 15-39.

12. Fakhouri F, Frémeaux-Bacchi V, Loirat C. Atypical hemolytic uremic syndrome: from the rediscovery of complement to targeted therapy. Eur J Intern Med 2013; 24 (6): 492-5. 
13. Fremeaux-Bacchi V, Fakhouri F, Garnier A, Bienaimé F, Dragon-Durey MA, Ngo S, et al. Genetics and outcome of atypical hemolytic uremic syndrome: a nationwide French series comparing children and adults. Clin J Am Soc Nephrol 2013;8 (4): 554-62.

14. Kavanagh D, Goodship TH. Atypical hemolytic uremic syndrome, genetic basis, and clinical manifestations. Hematology Am Soc Hematol Educ Program 2011; 2011 (1): 15-20.

15. Campistol JM, Arias M, Ariceta G, Blasco M, Espinosa M, Grinyó JM, et al. An update for atypical haemolytic uraemic syndrome: diagnosis and treatment. A consensus document. Nefrologia 2015; 35 (5): 421-47.

16. Coppo P, Bengoufa D, Veyradier A, Wolf M, Bussel A, Millot GA, et al. Severe ADAMTS13 deficiency in adult idiopathic thrombotic microangiopathies defines a subset of patients characterized by various autoimmune manifestations, lower platelet count, and mild renal involvement. Medicine (Baltimore) 2004; 83 (4): 233-44.

17. Cataland SR, Wu HM. Diagnosis and management of complement mediated thrombotic microangiopathies. Blood Rev 2014; 28 (2): 67-74.

18. Legendre CM, Licht C, Muus P, Greenbaum LA, Babu $\mathrm{S}$, Bedrosian C, et al. Terminal complement inhibitor eculizumab in atypical hemolytic-uremic syndrome. $\mathrm{N}$ Engl J Med 2013; 368 (23): 2169-81.

19. Cullinan N, Gorman KM, Riordan M, Waldron M, Goodship TH, Awan A. Case report: Benefits and challenges of long-term eculizumab in atypical hemolytic uremic syndrome. Pediatrics 2015; 135 (6): 1506-9.

20. Benamu E, Montoya JG. Infections associated with the use of eculizumab: recommendations for prevention and prophylaxis. Curr Opin Infect Dis 2016; 29 (4): 319-29.

21. Zuber J, Le Quintrec M, Morris H, Frémeaux-Bacchi V, Loirat C, Legendre C. Targeted strategies in the prevention and management of atypical HUS recurrence after kidney transplantation. Transplant Rev (Orlando) 2013; 27 (4): 117-25.

22. Licht C, Greenbaum LA, Muus P, Babu S, Bedrosian CL, Cohen DJ, et al. Efficacy and safety of eculizumab in atypical hemolytic uremic syndrome from 2-year extensions of phase 2 studies. Kidney Int 2015; 87 (5): 1061-73.

23. Ardissino G, Testa S, Possenti I, Tel F, Paglialonga F, Salardi S, et al. Discontinuation of eculizumab maintenance treatment for atypical hemolytic uremic syndrome: a report of 10 cases. Am J Kidney Dis 2014; 64 (4): 633-7.

24. Sepúlveda RA, Tagle R, Jara A. Novel Complement Factor $\mathrm{H}$ mutation, a case report of atypical hemolytic uremic syndrome. Case Reports in Internal Medicine 2017; 4 (2): 13-7.

25. Le Quintrec M, Zuber J, Moulin B, Kamar N, Jablonski $\mathrm{M}$, Lionet $\mathrm{A}$, et al. Complement genes strongly predict recurrence and graft outcome in adult renal transplant recipients with atypical hemolytic and uremic syndrome. Am J Transplant 2013; 13 (3): 663-75.

26. Snoeijs MG, Vink $H$, Voesten $N$, Christiaans $M H$, Daemen JW, Peppelenbosch AG, et al. Acute ischemic injury to the renal microvasculature in human kidney transplantation. Am J Physiol Renal Physiol 2010; 299 (5): 1134-40.

27. Fortin MC, Raymond MA, Madore F, Fugère JA, Pâquet $\mathrm{M}$, St-Louis G, et al. Increased risk of thrombotic microangiopathy in patients receiving a cyclosporin-sirolimus combination. Am J Transplant 2004; 4 (6): 946-52.

28. Zimmerhackl LB, Hofer J, Cortina G, Mark W, Würzner R, Jungraithmayr TC, et al. Prophylactic eculizumab after renal transplantation in atypical hemolytic-uremic syndrome. N Engl J Med 2010; 362 (18): 1746-8.

29. Hirt-Minkowski P, Schaub S, Mayr M, Schifferli JA, Dickenmann M, Frémeaux-Bacchi V, et al. Haemolytic uraemic syndrome caused by factor $\mathrm{H}$ mutation: is single kidney transplantation under intensive plasmatherapy an option? Nephrol Dial Transplant 2009; 24 (11): 3548-51.

30. Nester CM, Stewart Z, Myers D, Jetton J, Nair R, Reed A, et al. Pre-emptive eculizumab and plasmapheresis for renal transplant in atypical hemolytic uremic syndrome. Clin J Am Soc Nephrol 2011; 6 (6): 1488-94.

31. Sinha A, Gulati A, Saini S, Blanc C, Gupta A, Gurjar BS, et al. Prompt plasma exchanges and immunosuppressive treatment improves the outcomes of anti-factor $\mathrm{H}$ autoantibody-associated hemolytic uremic syndrome in children. Kidney Int 2014; 85 (5): 1151-60. 